Internist 2010 · 51:693-694

DOI 10.1007/s00108-010-2637-y

Online publiziert: 8. Mai 2010

(c) Springer-Verlag 2010

\author{
J. Mössner ${ }^{1}$. J.F. Riemann ${ }^{2}$ \\ ${ }^{1}$ Medizinische Klinik und Poliklinik II, Universitätsklinikum Leipzig AöR, Leipzig \\ ${ }^{2}$ Ehem. Direktor der Medizinischen Klinik C, Klinikum der Stadt Ludwigshafen
}

\title{
Dünndarm - Neue Wege in Diagnostik und Therapie
}

Der Dünndarm als der längste Abschnitt des Verdauungstrakts und bedeutendes Immunorgan des Menschen hat sich bis vor nicht allzu langer Zeit einer klaren direkten Betrachtung entzogen und war im Wesentlichen lediglich radiologischen indirekten Untersuchungsverfahren zugänglich. Das hat sich ganz entscheidend gewandelt. Neue bildgebende Verfahren der Radiologie und vor allem der Endoskopie haben die Diagnostik und in ersten Ansätzen auch die lokale Therapie von Erkrankungen dieses Organs grundsätzlich verändert. Hinzu kommt, dass sich, basierend auf wichtigen Erkenntnissen der Grundlagenforschung, neue Ansätze im Verständnis hormonaler und metabolischer Funktionen des Dünndarms ergeben haben.

\section{( Neue bildgebende Verfahren haben Diagnostik und lokale Therapie von Dünndarmerkrankungen grundsätzlich verändert}

Diesem neuen Erkenntnispfad trägt der erste Beitrag dieser Ausgabe von Der Internist Rechnung, der v. a. bislang unbekannte Funktionen der Enterozyten und der endokrinen Zellen des Dünndarms beschreibt. Funktionsvorgänge des Pankreas und der Gallenblase werden so auf molekularer Basis detaillierter verständlich. Neue Hormone wie das Ghrelin sowie neu aufgedeckte Funktionsweisen bekannter Peptide haben viel zum besseren Verständnis der Stoffwechselkontrolle einerseits sowie der Pathophysiologie zahlreicher Erkrankungen des Dünndarms beigetragen, sicherlich mit spannender Fortsetzung.

Das grundlagenwissenschaftliche Verständnis von Vorgängen im Dünndarm wird ganz erheblich durch die molekulare Bildgebung repräsentiert. Verschiedene Zelltypen des Dünndarms haben eine unterschiedliche molekulare Signatur, die sich sichtbar machen und damit zur Entdeckung von Veränderungen nutzen lässt. Die Verabreichung molekularer Marker, die durch Einbau in spezifische Oberflächenmoleküle auch metabolische Vorgänge sichtbar machen, hat bereits z. B. in der Nuklearmedizin die klare Darstellung endokriner Tumoren des Verdauungstrakts möglich gemacht und damit bereits Einzug in die klinische Routine gefunden. Die Endoskopie des Dünndarms nutzt die endogene wie die exogene Fluoreszenz, um so spezifische Zelltypen zu differenzieren und Stoffwechselvorgänge sichtbar zu machen. Hier steht die Entwicklung allerdings noch ganz am Anfang: Das, was man bis jetzt in diesem Zusammenhang sieht und zu verstehen beginnt, ist jedoch außerordentlich viel versprechend.

Im wahrsten Sinne Licht ins Dunkel des Dünndarms gebracht hat jedoch die Endoskopie, die inzwischen die komplette Untersuchung des Dünndarms möglich macht. Mit der Entwicklung der Doppelbzw. Singelballontechnik einerseits sowie der schnurlosen Kapselendoskopie andererseits haben außerordentlich präzise optische Verfahren Einzug in die Routinediagnostik des Verdauungstrakts, insbesondere auch des Dünndarms gehalten. Wir haben inzwischen gelernt, wie z. B. medikamentöse Veränderungen am Dünndarm aussehen. Wir können Erkran- 
kungen aus allen Dünndarmabschnitten nicht nur visuell, sondern auch bioptisch erfassen und auch hier therapeutische Interventionen wie Blutstillung, Polypektomie oder Fremdkörperentfernungen vornehmen. Die Technik ist heute so einfach geworden und standardisiert, dass sich die Dünndarmendoskopie nicht nur in Kompetenzzentren, sondern durchaus auch in vielen Krankenhäusern der Regelversorgung zu etablieren beginnt.

\section{(2) Die Dünndarmendoskopie beginnt sich auch in Krankenhäusern der Regelversorgung zu etablieren}

Aber auch die Radiologie hat sich entscheidend weiterentwickelt. Standen noch vor wenigen Jahren nur strahlenintensive Untersuchungen wie das Enteroklysma nach Sellink als Methoden zur Schleimhautdarstellung z. B. entzündlicher Dünndarmveränderungen zur Verfügung, sind heute die kontrastverstärkte Sonographie einerseits sowie die Magnetresonanztomographie des Dünndarms andererseits die Eckpfeiler der Dünndarmdiagnostik. Während die Wandbeurteilung mit beiden Verfahren gut möglich ist, bietet natürlich die MR-Enteroklyse eine Vielzahl von Zusatzinformationen, die v. a. bei entzündlichen Darmerkrankungen wie dem Morbus Crohn unverzichtbar sind. Der MR-Sellink gehört heute zur Standarduntersuchung zumindest in der Erstdiagnostik des Morbus Crohn und hat die strahlenintensiven Untersuchungsverfahren abgelöst.

Nicht nur diagnostische Verfahren lassen Erkrankungen besser erkennen, auch neue therapeutische Ansätze haben zu einer wesentlich besseren Therapie und damit auch zu einer deutlich besseren Prognose einiger wichtiger Dünndarmerkrankungen geführt. Beispielsweise ist die antibiotische Therapie der Whippleschen Erkrankung heute standardisiert und bietet beste Aussichten auf lang anhaltende Remissionen. Neue Biologika greifen in die Signaltransduktionswege von Entzündungsvorgängen ein und bieten somit als ein mögliches therapeutisches Ziel auch die Schleimhautheilung, was bis dahin in diesem Umfang nicht erreicht werden konnte. Auch Probiotika, lange verkannt in ihrer Bedeutung, haben durch ihre speziellen Wirkstoffe in der Therapie so mancher Durchfallerkrankung einen wesentlichen Zusatznutzen geliefert.

Der Gastroenterologe kann mit diesen neuen Entwicklungen schon seit längerer Zeit umgehen und sie einsetzen. Ziel dieses Heftes ist es, auch dem Internisten allgemein ebenso wie den Internisten mit anderen Schwerpunkten die Fortschritte im Bereich von Erkrankungen des Dünndarms aufzuzeigen. Der Internist sollte heute wissen, wie er die verschiedenen diagnostischen und therapeutischen Möglichkeiten in seine differenzialdiagnostischen und -therapeutischen Überlegungen einbauen kann, wie sie zu werten sind und wann es nötig ist, den Spezialisten hinzuzuziehen. Er sollte auch sein Verständnis für die Grundlagenforschung in diesem Bereich weiterentwickeln, die letztlich in der Zukunft neue zielgerichtete Therapien möglich macht.

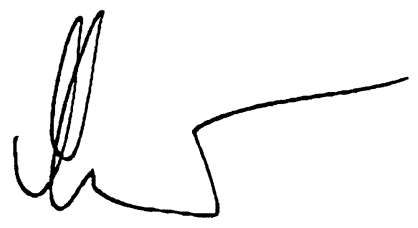

Prof. Dr. Joachim Mössner

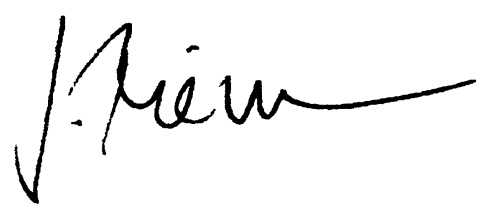

Prof. Dr. Jürgen F. Riemann

\section{Korrespondenzadresse}

Prof. Dr. J.F. Riemann

Ehem. Direktor der Medizinischen Klinik C, Klinikum der Stadt Ludwigshafen Bremserstraße 79, 67063 Ludwigshafen riemannj@garps.de

\section{Pankreatitis: Duodenum operativ entfernen?}

Für eine klinische Studie der Universitätsklinik Heidelberg werden Patienten mit chronischer Pankreatitis gesucht. Im Mai 2009 startete die von der Deutschen Forschungsgemeinschaft DFG geförderte "ChroPac"- Studie des Studienzentrums der Deutschen Gesellschaft für Chirurgie an der Chirurgischen Universitätsklinik Heidelberg. Neben Heidelberg nehmen 12 weitere Kliniken im In- und Ausland an der Untersuchung teil. Im Rahmen der Studie werden zwei gängige Operationsverfahren zur Behandlung von Pankreatitis miteinander verglichen: Bei der Whipple Operation werden neben dem entzündeten Kopf des Pankreas auch Teile des Duodenums und des Magens, die Gallenblase und ein Teil des Gallenganges entfernt. Im zweiten Verfahren bleiben Magen und Duodenum erhalten. Der Pankreaskopf wird ausgeschält, und die Verengungen am Gallen- und Pankreasgang werden beseitigt. Beide Verfahren sind etablierte und gleichwertige Operationsmethoden mit vergleichbaren Risiken und Nebenwirkungen. Ziel der doppelblind angelegten Studie ist es herauszufinden, welches der beiden Verfahren langfristig bessere Ergebnisse zeigt und den Patienten damit eine höhere Lebensqualität bietet.

Alle an der Studie teilnehmenden Patienten werden im Anschluss an die Operation über zwei Jahre nachuntersucht. Interessierte, nicht voroperierte Patienten können sich unter 06221-56-37728 über das für Sie nächstgelegene Studienzentrum informieren.

Literatur: Diener MK, Knaebel HP, Heukaufer $C$ et al (2007) A systematic review and meta-analysis of pylorus-preserving versus classical pancreaticoduodenectomy for surgical treatment of periampullary and pancreatic carcinoma. Annals of Surgery 245(2):187-200

Quelle: Universitätsklinik Heidelberg, www.klinikum.uni-heidelberg.de 